\title{
Plantas medicinales que se venden en el mercado El Río, Camagüey, Cuba
}

\author{
Medicinal plants sold at the El Río Market, Camagüey, Cuba
}

\author{
Daimy Godínez-Caraballo ${ }^{1 *}$ y Gabriele Volpato² \\ ${ }^{1}$ Centro de Investigaciones de Medio Ambiente de Camagüey, Cuba. Calle Cisneros 105 altos, e/Pobre y Ángel, 70100 Camagüey, Cuba. \\ ${ }^{2}$ Universidad de Wageningen, Wageningen, Países Bajos. \\ *Correspondencia: daimy@cimac.cu
}

\begin{abstract}
Resumen. Con la información obtenida de las plantas medicinales vendidas en el mercado El Río, Camagüey, Cuba, se registró un total de 184 especies pertenecientes a 69 familia de plantas vasculares. La familia más importante fue Fabaceae s.l. con 13 especies, seguida por Lamiaceae con 12 y Asteraceae con 8. Más de 90 indicaciones medicinales en general fueron recogidas para tratar un amplio rango de enfermedades y dolencias. El mayor número de especies y aplicaciones fueron reportadas para desórdenes del sistema digestivo, sistema musculoesquelético (reumatismo y contusiones), problemas renales y trastornos ginecológicos. Las partes de las plantas más usadas fueron la hoja (42.9\%) y el fruto (22.3\%). Los remedios se preparan principalmente como decocción (51.6\%) e infusión (39.1\%). Los datos recolectados muestran el conocimiento y uso de la medicina herbolaria por parte de la población camagüeyana para tratar varias enfermedades.
\end{abstract}

Palabras clave: ventas, plantas medicinales, mercado, Camagüey, Cuba.

\begin{abstract}
Information regarding medicinal plants sold in the El Río Market, Camagüey, Cuba, revealed 184 species belonging to 69 vascular plant families. The most important family was Fabaceae s.l. with 13 species, followed by Lamiaceae with 12, and Asteraceae with 8 . More than 90 general medicinal indications were recorded to treat a wide range of illnesses and ailments. The highest number of species and applications were reported for digestive system disorders, the musculoskeletal system (rheumatism and the complex of contusions), kidney and other urological problems, and gynecological disorders. The part of the plant most frequently used was the leaf (42.9\%) and the fruit (22.3\%). The remedies were mainly prepared as a decoction $(51.6 \%)$ and an infusion $(39.1 \%)$. The present data document the knowledge of herbal medicine by villagers of Camagüey for to treat health problems.
\end{abstract}

Key words: on sale, medicinal plants, market, Camagüey, Cuba.

\section{Introducción}

En Cuba, los usos de las plantas con fines alimenticios y medicinales representan el resultado histórico de la interacción entre los grupos étnicos dentro de la cultura cubana, y entre éstos y el medio ambiente isleño (Esquivel y Hammer, 1992; Núñez y González, 1999; Hernández y Volpato, 2004; Volpato y Godínez, 2004).

En Camagüey, como en toda Cuba, el uso de las plantas medicinales en vez de los medicamentos farmacéuticos está muy extendido y diversas pudieran ser las causas que llevan a esto. Diferentes autores han listado las plantas útiles al hombre en esta provincia (Méndez et al.,1986; Godínez y Rosete, inédito; entre otros), y dentro de ellas las medicinales (Barreto et al., 1992; Beyra et al., 2004).

Recibido: 31 octubre 2006; aceptado: 24 mayo 2007
Debe destacarse que los mercados son espacios que constituyen reservorios de germoplasma y contribuyen a la preservación de la diversidad a través del uso (Shagarodsky et al., 2003). Pero aún son escasos los trabajos en el país que hablan de ello (Fernández et al., 1999; Shagarodsky et al., 2001) y la mayor parte de los artículos realizados están dirigidos a plantas medicinales (Fuentes y Granda, 1988; Hernández, 2000).

En Camagüey, de los 5 mercados existentes, el más importante es El Río, por registrarse ahí la venta más alta y mantener una oferta variada de productos agropecuarios, en algunos casos con precios,más bajos que en los restantes; así también, porque está ubicado en uno de los lugares más céntricos de la ciudad y el local presenta las mejores condiciones para el acto de compra-venta (Nasser, 2005).

Los objetivos del trabajo fueron: 1) listar las plantas medicinales que se venden en el mercado El Río de 
Camagüey y que son utilizadas por la población de esta ciudad, y 2) obtener información sobre las dolencias que curan, las partes de la planta que se usan y su forma de preparación.

\section{Materiales y métodos}

El trabajo se desarrolló en el mercado El Río de la ciudad de Camagüey, el cual ocupa una céntrica posición dentro del municipio, cabecera de la provincia del mismo nombre (Fig. 1), y se caracteriza por la gran afluencia de vendedores y compradores así como por la variedad y cantidad de productos que ahí se ofertan.

La información se tomó mediante observación directa del producto en las tarimas, las tablillas que muestran la oferta del mismo y mediante entrevistas abiertas, semiestructuradas, que se hicieron a los vendedores de plantas medicinales, siguiendo la metodología de Alexiades (1996).

Los materiales colectados se herborizaron utilizando los métodos tradicionales, se identificaron y ubicaron taxonómicamente consultando las obras de León (1946), León y Alain (1951), Alain (1953, 1957, 1964), Catasús (1997), Bässler (1998), Barreto (1999), Rodríguez (2000), Gutiérrez (2000, 2002), Albert (2005). Los ejemplares fueron depositados en el herbario del Centro de Investigaciones de Medio Ambiente de Camagüey (HACC).

\section{Resultados}

A partir de las entrevistas realizadas y la observación de las listas de venta en el momento del estudio, se compilaron 184 taxones infragenéricos distribuidos en 163 géneros pertenecientes a 69 familias botánicas que se expenden en el mercado El Río de la ciudad de Camagüey. Se elaboró una lista de esas plantas (en orden alfabético por familia; Apéndice 1). Para cada una de las especies se brinda: nombre botánico, nombre (s) local (es) y observaciones sobre su uso y modo de preparación.

Las familias más importantes por el número de especies fueron Fabaceae s.l. (13), Lamiaceae (12), Asteraceae (8). Siguen, en orden decreciente, Poaceae, Myrtaceae y Apiaceae con 7, Rubiaceae, Rutaceae y Solanaceae con 6 y Euphorbiaceae y Cucurbitaceae con 5 cada una. Estas 11 familias representaron el $44.5 \%$ del total de taxones vendidos, las restantes poseen sólo de 1 a 4 especies.

Se listaron 193 nombres locales de todas las plantas medicinales vendidas.Se recopilaron más de 90 indicaciones medicinales generales para tratar un amplio rango de enfermedades y dolencias. El mayor número
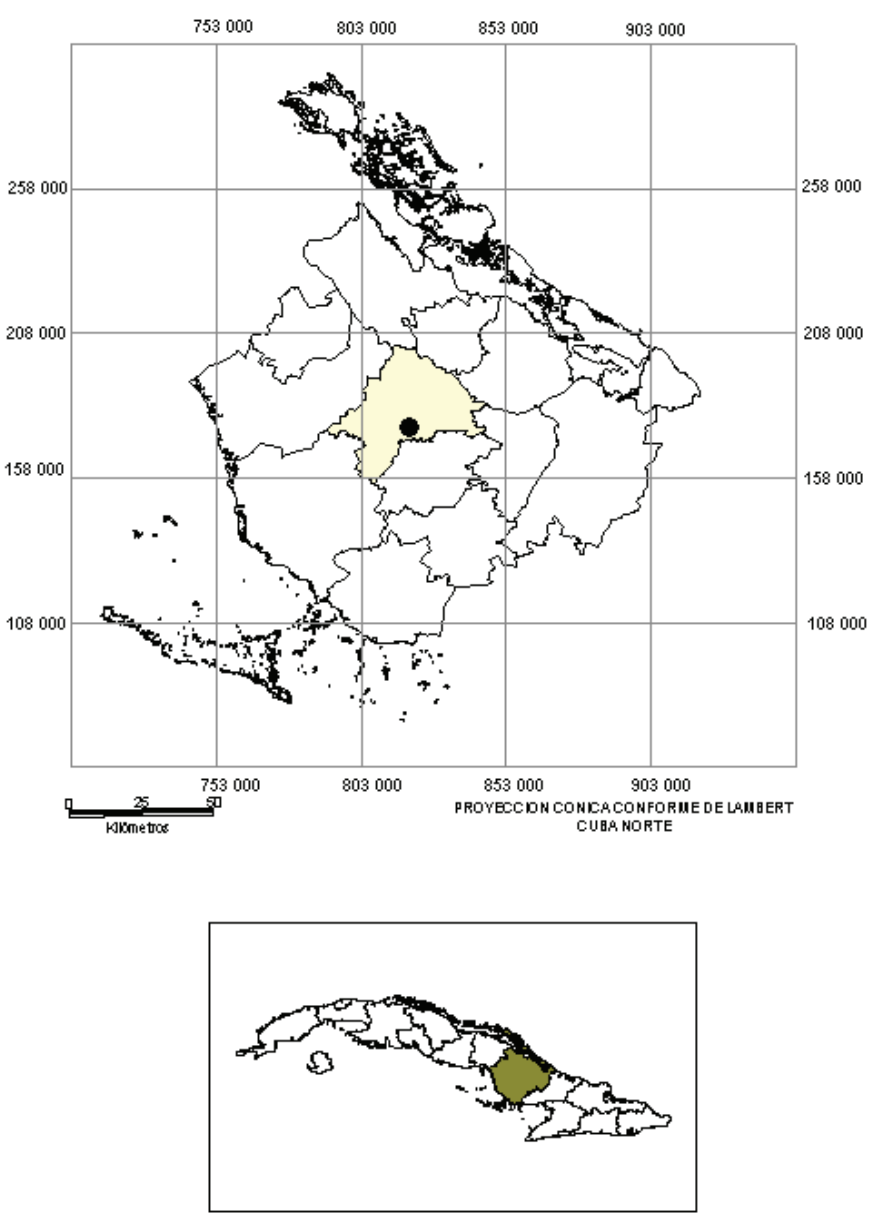

Figura 1. Ubicación geográfica del área de estudio.

de especies (65), que representan el $35.3 \%$ del total de taxones, son vendidas para curar los desórdenes del sistema digestivo, principalmente las afecciones gastrointestinales, parásitos y diarreas, incluidas las úlceras y problemas en el hígado, con 5 y 10 especies registradas; en segundo lugar estuvieron las afecciones respiratorias y problemas renales, con 25 y 14 especies respectivamente.

Del análisis de los usos medicinales de cada taxon, se detectaron conceptos y percepciones muy interesantes, como por ejemplo, especies calientes: Bursera simaruba (almácigo), nutracéuticas: Acrocomia aculeata (corojo), Sesamum orientale (ajonjolí) y aquellas que curan el empacho: Plantago major (llantén), Laurel nobilis (laurel) y Blighia sapida (seso vegetal).

Las hojas $(42.9 \%)$ y frutos $(22.3 \%)$ fueron las partes de la planta más usadas como medicinales, seguidas por la corteza (16.3\%) y semillas (14.6\%) (Fig. 2).

Se detectó que la mayoría de las especies se venden 


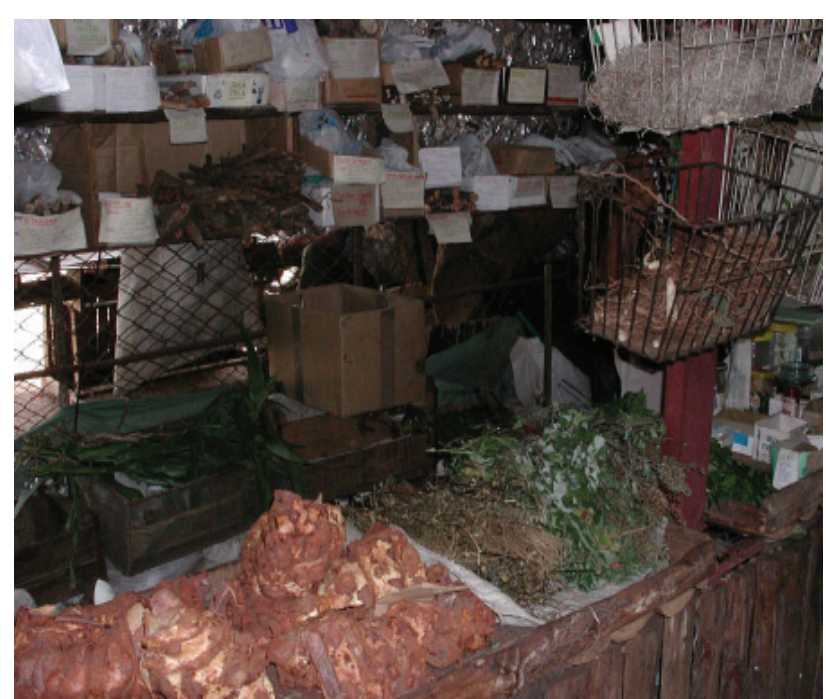

Figura 2. Hojas, frutos, cortezas y semillas son las partes más usadas con fines medicinales.

frescas, mientras que las partes como semillas, raíces, cortezas y resinas de algunas plantas, para algún uso específico medicinal, se venden secas (Fig. 3). La mayor parte de los remedios que se ofrecen son para prepararse como decocción (51.6\%), infusión (39.1\%), jugos (13.0\%) y cataplasmas $(8.7 \%)$.

\section{Discusión}

De lo anterior se evidencia que en el mercado El Río de la ciudad de Camagüey, además de los productos agropecuarios que se ofertan, se comercializa una gran diversidad de plantas medicinales, las cuales se brindan para tratar un amplio espectro de enfermedades y dolencias. El número de especies vegetales que se expenden constituye el $92 \%$ de las 200 plantas utilizadas como medicinales por la población cubana, según registros de Martínez et al. (1987) al hablar de la importancia de la etnobotánica en Cuba. Beyra et al. (2004) registran 111 especies de uso local y popular en 7 comunidades de la provincia camagüeyana, y en el presente estudio se encontró que solamente en una pequeña área se expenden 184, lo que explica por qué la cifra de taxones medicinales que se venden puede fluctuar en dependencia de la época del año y/o demanda de determinado producto (Rolando Pérez, com. pers.) Los vendedores buscan y reciben plantas medicinales de todo el país, sobre todo de la zona oriental cuando éstas no se encuentran en Camagüey; así también, las compran a personas que cultivan jardines y huertos (Anita Martínez, com. pers.).

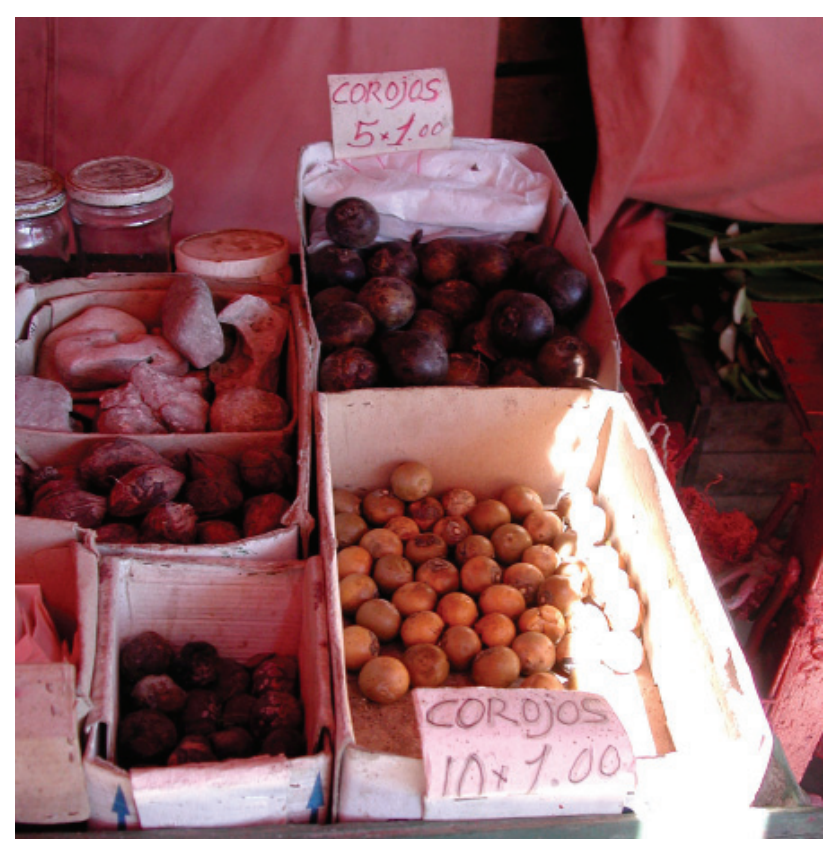

Figura 3. Venta de semillas, raíces, cortezas y resinas de uso medicinal.

Coincide el hecho de que las familias con mayor riqueza específica son las más importantes dentro de la flora cubana (Alain, 1958;, Acevedo, 1991 y Vales et al., 1998;). Incluso otros estudios etnobotánicos en Cuba han registrado estas familias por su relevante número de especies (Barreto et al., 1992; Scull et al., 1998; Hernández, 2000; Beyra et al., 2004; Hernández y Volpato, 2004).

Cada especie tiene al menos un nombre local o vulgar. Muchas de ellas se conocen principalmente por su denominación vernácula, la cual se utiliza con amplitud entre todos los informantes (vendedores) y compradores. Esta uniformidad entre los vendedores, en lo que a nombres comunes respecta, probablemente indica que de forma general los recursos medicinales y remedios para los que se utilizan son bien conocidos.

El uso de las plantas para curar diferentes problemas del aparato gastrointestinal es la categoría más utilizada en ésta y otras ciudades de Cuba, como es el caso de Santiago, donde existen variedades de mixturas que desempeñan un papel importante en la medicina tradicional (Hernández y Volpato, 2004).

Los términos caliente o frío, se refieren al sistema humoral en el cual se subdividen todas las especies de plantas y enfermedades (Quinlan y Quinlan, 2006). En este estudio, el hecho de considerar medicamentos con propiedades calientes para tratar enfermedades frías o viceversa, confirman lo planteado por Fuentes (1984) 
y Nuñez (1999) sobre algunas de las características de la medicina tradicional popular en Cuba, como lo es la dicotomía frío-caliente; ampliamente difundida en los sistemas etnomédicos de Latinoamerica y Afrocaribe (Laguerre, 1987; Foster, 1994).

También en Dominica, muchos remedios para las condiciones calientes o frías consisten en ingerir aquellos alimentos con propiedades opuestas para contrarrestar las causas de la enfermedad (Quinlan y Quinlan, 2006).

Un alimento nutracéutico es como decir nutritivo. Las personas que basan su alimentación bajo una dieta macrobiótica consumen el ajonjolí (Sesamum orientale) como una comida nutracéutica por ser fuente de proteínas (Gastón, com.pers.). Por otro lado, se conoce que en Cuba muchos de los platos típicos de origen africano incluyen especies depurativas y nutritivas (por ej., el corojo),que la población afrocubana considera alimentos secundarios y para época de escasez (Valdés, 1987; Esquivel et al., 1992).

En muchas partes de Cuba, estas palmas espinosas (corojo) se usan como sustituto del coco (Cocos nucifera) $\mathrm{y}$ son apreciadas por las propiedades medicinales $\mathrm{y}$ alimenticias de sus frutos (Leiva, 2001).

En Cuba la palabra empacho es el término que generalmente se emplea para denominar una indigestión. El prú es una bebida refrescante cubana que ayuda al proceso digestivo y cura los empachos, limpiando las paredes del estómago (Volpato y Godínez, 2004; Hernández y Volpato, 2004). Muchos informantes describen el empacho como algo parecido a una bola o comida indigesta dentro del estómago (Volpato y Godínez, 2006).

Hernández (2000) plantea que en la ciudad de Santiago de Cuba la costumbre de la población de consumir plantas frescas conlleva a una estrecha coordinación entre colectores y expendedores para evitar pérdidas tanto por exceso como por defecto. Lo cual se evidencia en este estudio.

\section{Agradecimientos}

Al MsC. Eddy Martínez Quesada por su valiosa y desinteresada ayuda en el diseño de los mapas de ubicación del área.

\section{Literatura citada}

Acevedo, P. 1991. Angiosperms in the Greater Antilles. Flora of the greater Antilles Newsletter 1:3-5.

Alain, Hno. 1953. Plantas tintóreas y curtientes vegetales. Flora de Cuba. Contribuciones Ocasionales del Museo de Historia Natural Colegio La Salle. La Habana 3. 502 p.
Alain, Hno. 1957. Flora de Cuba. Contribuciones Ocasionales del Museo de Historia Natural Colegio La Salle. La Habana 4. $556 \mathrm{p}$.

Alain, Hno. 1958. La flora de Cuba: sus principales características, su origen probable. Revista de la Sociedad Cubana de Botánica 15: 36-59.

Alain, Hno. 1964. Flora de Cuba. Publicaciones de la Asociación para el Estudio de las Ciencias Biológicas (La Habana) 5. $362 \mathrm{p}$.

Albert, D. 2005. Meliaceae. In Flora de la República de Cuba. Serie A. Plantas vasculares. fascículo 10(5), W. Greuter y R. Rankin Rodríguez (eds.). Gantner, Ruggell, Liechtenstein. $44 \mathrm{p}$.

Alexiades, M.N. 1996. Collecting ethnobotanical data: an introduction to basic concepts and techniques. In Selected guidelines for ethnobotanical research: a field manual, M.N. Alexiades (ed.). The New York Botanical Garden, New York. p. 53-94.

Barreto, A., E. Pérez, A. Beyra y N. Enríquez. 1992. Flora angiospérmica de la Provincia Camagüey, Cuba. Acta Botanica Cubana 91: 1-105

Barreto, A. 1999. Las leguminosas (Fabaceae) de Cuba, I. Subfamilia Caesalpinioideae. Collectanea Botanica 24:6-148.

Bässler, M. 1998. Mimosaceae. In Flora de la República de Cuba. Serie A, Plantas vasculares, fascículo 2. Koeltz, Königstein. $202 \mathrm{p}$.

Beyra, A., M.C. León, E. Iglesias, D. Ferrándiz, R. Herrera, G. Volpato, D. Godínez, M. Guimarais y R. Álvarez. 2004. Estudios etnobotánicos sobre plantas medicinales en la provincia de Camagüey (Cuba). Anales del Jardín Botánico de Madrid 61:185-204.

Catasús, L. 1997. Las gramíneas (Poaceae) de Cuba I. Fontqueria XLVI: 1-259.

Esquivel, M. y K. Hammer. 1992. The Cuban homegarden 'conuco': a perspective environment for evolution and in situ conservation of plant genetic resources. Genetic Resources and Crop Evolution 39:9-22.

Esquivel, M., V. Fuentes, C. Martínez, J. Martínez y K. Hammer. 1992. The African influence from an ethnobotanical point of view. In "...y tienen faxones y fabas muy diversos de los nuestros...." Origin, evolution and diversity of Cuban plant genetic resources, vol. 1, K. M. Hammer Esquivel and H. Knüpffer (eds.). Institut für Pflanzengenetik und Kulturpflanzenforschung, Gatersleben. p. 83-109.

Fernández, M., C., Martínez, S. Rosete y J. I. Martínez.1999. Agromercados en Cuba: reservorios de germoplasma. Acta Botanica Cubana 125:1-8.

Foster, G. M. 1994. Hippocrates' Latin American legacy. Humoral medicine in the New World. Gordon and Breach, Langhorne, Pennsylvania.

Fuentes, V. 1984. Sobre la medicina tradicional en Cuba. Boletín de Reseña de Plantas Medicinales 10. Centro de Información y Documentación Agropecuaria, La Habana. 39 p.

Fuentes, V. y M. Granda.1988. Estudios sobre la medicina tradicional en Cuba I. Revista Plantas Medicinales (Cuba) 2:25-46.

Gutiérrez, J. 2000. Flacourtiaceae. In Flora de la República de Cuba Serie A. Plantas vasculares, fascículo 5, W. Greuter y 
R. Rankin Rodríguez (eds.). Koeltz, Königstein. 76 p.

Gutiérrez, J. 2002. Sapotaceae. In Flora de la República de Cuba Serie A. Plantas vasculares, fascículo 6, W. Greuter y R. Rankin Rodríguez (eds.). Koeltz, Königstein. 59 p.

Hernández, J. 2000. Plantas que se expenden en las yerberías de la ciudad de Santiago de Cuba. Biodiversidad de Cuba centro-oriental 5: 65-72.

Hernández, J.y G. Volpato. 2004. Herbal mixtures in the traditional medicine of eastern Cuba. Journal of Ethnopharmacology 90:293-316.

Laguerre, M. 1987. Afro-Caribbean folk medicine. Bergin and Garvey, South Hadley, Massachusetts. viii+120 p.

Leiva, Á. 2001. Cuba y sus palmas. Gente Nueva, La Habana. 73 p.

León, Hno. 1946. Flora de Cuba. Contribuciones Ocasionales del Museo de Historia Natural Colegio La Salle. La Habana 1. $441 \mathrm{p}$.

León, Hno. y Hno. Alain. 1951. Flora de Cuba. Contribuciones Ocasionales del Museo de Historia Natural Colegio La Salle. La Habana 10. 456 p.

Martínez, C., P. Herrera, R. Oviedo y E. Moreno. 1987. Importancia de la etnobotánica en Cuba. Reporte de Investigación del Instituto de Ecología y Sistemática 20: $1-17$.

Méndez, I. E., R. Trujillo y V. Martínez. 1986. Inventario florístico del Cerro Tuabaquey (Sierra de Cubitas, Camagüey). Revista Jardín Botánico Nacional 7: 67-78.

Nasser, M. M. 2005. El mercado agropecuario. Sus virtudes y defectos. Una aproximación al problema. Tesis, Facultad de de Ciencias Agropecuarias, Universidad de Camagüey. 50 p.

Núñez, N. 1999. Algunas concepciones alimentarias de los cubanos. Revista Cubana de Alimentación y Nutrición 13:46-50.

Núñez, N. y E. González. 1999. Antecedentes etnohistóricos de la alimentación tradicional en Cuba. Revista Cubana de
Alimentación y Nutrición 13: 145-150.

Quinlan, M. B. y R. J. Quinlan. 2006. Balancing the system: humoral medicine and food in the commonwealth of Dominica, chapter 8. In Eating and healing: traditional food as medicine, A. Pieroni and L. L. Price (eds.). Haworth, New York. p. 197-212.

Rodríguez, A. 2000. Sterculiaceae. In Flora de la República de Cuba Serie A. Plantas vasculares, fascículo 3. Koeltz, Königstein. 68 p.

Shagarodsky, T., Z. Fundora, L. Castiñeiras, O. Barrios, V. Moreno, L .Fernández, V. Fuentes, R. Cristóbal, C. Giraudy, M. García, P. Sánchez, V. González, F. Hernández y A. Valiente. 2001. Diversidad de productos agrícolas en los sistemas de fincas y su presencia en el mercado. Cuba. Agricultura Orgánica 7:24-28.

Shagarodsky, T., V. Fuentes, O. Barrios, L. Castiñeiras, Z. Fundora, P. Sánchez, L. Fernández, R. Cristóbal, M. García y C. Giraudy. 2003. Diversidad de especies alimenticias en tres mercados agrícolas de la Habana, Cuba. Agronomía Mesoamericana 14:27-39.

Scull, R., M. Miranda y R. Infante. 1998. Plantas medicinales de uso tradicional en Pinar del Río. Estudio etnobotánico. I. Revista Cubana de Farmacia 32: 57-62

Valdés, S. 1987. Las lenguas del África subsahariana y el español de Cuba. Academia de Ciencias de Cuba, La Habana.

Vales, M., A. Álvarez, L. Montes y A. Ávila (eds.). 1998. Estudio nacional sobre la diversidad biológica en la República de Cuba. CESYTA, Madrid.

Volpato, G. y D. Godínez. 2004. Ethnobotany of pru, a traditional Cuban refreshment. Economic Botany 58: 381-395.

Volpato, G. y D. Godínez. 2006. Medicinal foods in Cuba: Promoting health in the household, chapter 9. In Eating and healing: traditional food as medicine, A. Pieroni and L. L. Price (eds.). Haworth, New York. p. 213-236.

Apéndice 1. Lista de plantas medicinales que se venden en el Mercado El Río, Camagüey, Cuba.

\section{ACANTHACEAE}

Blechum brownei Juss. (mazorquilla)

La decocción de la parte aérea se toma como diurético y febrífugo.

Justicia pectoralis Jacq. Tilo

Se informa que la decocción de la parte aérea se usa como pectoral y sedativo, para calmar los nervios.

\section{ALOACEAE}

Aloe vera L. (sábila)

De las hojas frescas se extrae el mesofilo, el cual se corta en pequeñas piezas (cristalitos de sábila) que se ingieren por día para el tratamiento de las afecciones del hígado y como laxante.Estas misma piezas son usadas vía anal como supositorios para curar las hemorroides y vía vaginal en el tratamiento de infecciones vaginales.También en cataplasmas se aplican sobre quemaduras y úlceras. 
La pulpa del fruto se emplea como astringente y antidiarreico, pectoral y útil contra los esputos de sangre. Las semillas tostadas son consideradas un poderoso afrodisíaco. La infusión de las hojas se brinda como antidiarreico.

Mangifera indica L. (mango)

El fruto se come para tratar las afecciones gastrointestinales. La decocción de las semillas es vermífuga y la de la cáscara del fruto es anticatarral. También para tratar dolor de dientes y lavar heridas infectadas.

Spondias mombin L. (jobo)

La infusión de la corteza se toma como astringente y para lavar las heridas infectadas. El fruto ingerido en altas dosis es un poderoso emético. La decocción de la corteza se recomienda para tratar las hemorroides.

Spondias purpurea L. (ciruela)

El fruto crudo es laxante. La decocción de las hojas si se aplica localmente sirve para combatir las afecciones de la piel.

ANNONACEAE

Annona cherimolia L. (chirimoya)

El batido del fruto con leche y azúcar se bebe para tratar la anemia. La infusión de las hojas se toma como antidiarreico. Annona muricata L. (guanábana)

La infusión de las hojas se registra como anticatarral y útil en la cura de las afecciones respiratorias.

Annona squamosa L. (anón)

La infusión de las hojas se emplea para curar los resfriados estomacales, y mezclada con yerbabuena y mejorana como anticatarral. La decocción de la corteza, las hojas, y la cáscara del fruto se utiliza en la cura de las afecciones gastrointestinales.

\section{APIACEAE}

Anethum graveolens L. (eneldo)

La infusión de la planta entera se recomienda como diurético y para curar los cólicos gastrointestinales. La decocción del fruto se emplea como galactógeno.

Apium graveolens L. (apio)

El jugo de la parte aérea con miel se toma para curar las afecciones del hígado. Y también como antianémico.

Coriandrum sativum L. (cilantro)

La decocción de la parte aérea es anticatarral, antihipertensivo y antidiabético. La decocción de la raíz es abortiva. La infusión de la parte aérea se toma como estomáquico y para eliminar los gases intestinales. La decocción del fruto se emplea en para tratar el dolor de cabeza y las fiebres intermitentes.

Cuminum cyminum L. (comino)

La decocción de las semillas es buena como anticatarral y los buches de la misma para el dolor en los dientes.

Foeniculum vulgare L. (hinojo)

La infusión de la parte aérea se toma como galactógeno, digestivo, carminativo y antiespasmos.

Petroselinum crispum (Mill.) Nym. (perejil)

El jugo de las hojas y tallos mezclado con miel se toma para tratar la anemia.

Pimpinella anisum L. (anís estrellado)

La infusión de los frutos se toma como galactógeno y antiespamódico, pero puede causar irritaciones gastrointestinales.

\section{ARECACEAE}

Acrocomia aculeata (Jacq.) Lodd (corojo)

El fruto se considera nutracéutico, y también con propiedades refrescantes y diuréticas. La mantequilla que se hace de las semillas se aplica como antiinflamatorio en las infecciones de articulaciones. El aceite que se extrae de las semillas fortalece y preserva el cabello.

Cocos nucifera L. (coco)

El endospermo de naturaleza líquida (agua de coco) se bebe como diurético. La mantequilla hecha de las semillas se aplica de forma externa como antiinflamatorio en afecciones de la piel, e ingeridas como laxante. La leche extraída de los frutos cura la inflamación de las manos. La decocción de la raíz se emplea como diurético.

Roystonea regia (H. B. K.) O. F. Cook (palma real)

La raíz se emplea como diurético y es buena contra la diabetes, infecciones internas y para tratar los cálculos renales. 


\section{ASTERACEAE}

Ambrosia artemisifolia L. (artemisa)

La infusión de las hojas se toma como vermífugo, y en fricciones externas para tratar los dolores reumáticos.

Bidens pilosa L. (romerillo)

La infusión de la parte aérea ayuda a la digestión. Las inhalaciones alivian la bronquitis y dolor de garganta. El cabello se fortalece si se lava con la decocción de esta planta.

Lactuca sativa L. (lechuga)

La infusión de los tallos se toma para combatir el insomnio y como sedativo.

Matricaria recutita L. (manzanilla)

Una infusión de la parte aérea se toma como carminativa, estomáquica, aperitivo y para lavar las infecciones de la piel. Se informa que la raíz combate el dolor de muelas.

Pluchea carolinensis (Jacq.) G. Don. (salvia de playa)

Las hojas se aplican en la frente y en la sien para combatir el dolor de cabeza. La decocción de las hojas y los tallos se bebe como digestivo, febrífugo y las alteraciones menstruales. El jugo se toma macerado con café como anticatarral y para curar los dolores de garganta y ronquera.

Pseudelephantopus spicatus (Juss.) Rohr. (lengua de vaca)

La decocción de la raíz se considera como un poderoso febrífugo. El cataplasma hecho de las hojas es antiinflamatorio. Tagetes erecta L. (flor de muerto)

La decocción de las flores se emplea como pectoral y anticatarral. La infusión de las hojas y flores, como vermífugo.

Xanthium strumarium L. (guizazo de caballo)

La decocción de la raíz se considera buena para los cálculos y cólicos renales, como diurético y purificador de la sangre; la de las hojas, como antiséptico.

BASELLACEAE

Basella rubra L. (espinaca de malabar)

Las hojas se aplican externamente en forma de cataplasma para tratar las inflamaciones. La raíz se brinda para disolver los tumores externos e internos.

Boussingaultia leptostachys Moq. (yuca hiedra)

El rizoma se aplica externamente con agua caliente en casos de huesos rotos. La infusión del rizoma y las hojas se bebe para las afecciones del hígado, dolor de dientes y para tratar la taquicardia.

\section{BIGNONIACEAE}

Salpianthus purpurascens Hook.et Arn. (nitro)

La decocción de la parte aérea se toma como diurético y para el tratamiento de los cálculos y cólicos renales.

Crescentia cujete L. (güira)

El jugo de las flores se aplica localmente para el dolor de oído. Con la pulpa del fruto, ron y miel se hace un jarabe llamado miel de güira de propiedades anticatarrales, anticancerosas, expectorante y cicatrizante, además se recomienda para limpiar la matriz en el caso de las mujeres que deseen tener un embarazo.

Mirabilis jalapa L. (maravilla)

La decocción de la raíz se considera un poderoso laxante y vermífugo.

Tecoma stans (L.) H.B.K (sáuco amarillo)

La decocción de hojas y tallos es buena en la diabetes, mientras que la de la raíz es un diurético poderoso.

\section{BIXACEAE}

Bixa orellana L. (bija)

La cáscara de la semilla se aplica para curar las infecciones superficiales y mordeduras de insectos. La infusión de las hojas es astringente, febrífuga, diurética y ligeramente afrodisíaca. Se aplica en forma de cataplasma para el dolor de cabeza.

\section{BOMBACACEAE}

Ceiba pentandra (L.) Gaertn. (ceiba)

La decocción de la corteza se considera un diurético y aperitivo eficaz. La infusión de las flores se toma como 
antiinflamatorio. El aceite obtenido de las semillas elimina las infecciones de la piel.

BORAGINACEAE

Cordia gerascanthus L. (varia)

Una infusión de las hojas se toma como anticatarral y contra las afecciones respiratorias. La decocción de la raíz es útil en el tratamiento de epilepsia.

Heliotropium angiospermum Muray (alacrancillo)

La infusión de las hojas es buena contra los cálculos renales. El jugo de las hojas es bueno para los eczemas y en gárgaras para curar las afecciones de la garganta.

Varronia globosa (Jacq.) HBK. ssp. humilis (Jacq.) Borhidi (yerba de la sangre)

La decocción de la parte aérea se administra internamente como purificador de sangre y como hemostático si se aplica externamente en forma de lavado.

BRASSICACEAE

Lepidium virginicum L. (mastuerzo)

La decocción de la parte aérea se considera un diurético poderoso, antidiabético y antireumático.

BROMELIACEAE

Ananas comosus L. (piña)

El jugo de la fruta es bueno para hacer gárgaras y tratar el dolor de garganta.

Bromelia pinguin L. (piña de ratón)

El jugo de la fruta, macerado con el azúcar, se considera un vermífugo poderoso.

Tillandsia sp. (curujey)

La infusión de las hojas mezcladas con gandul y yerba Paraná se recomienda para tratar la giardiasis. Las hojas aplastadas mezcladas con aceite se aplican localmente para curar las hemorroides.

Tillandsia usneoides L. (guajaca)

La decocción de la planta entera fortalece el cabello y se recomienda como antiasmático y antihipertensivo.

BURCERACEAE

Bursera graveolens Triana et Planch (sasafrás)

La corteza macerada en alcohol se utiliza en fricciones para aliviar las articulaciones. La decocción de la parte aérea y la resina se toma para curar las afecciones gastrointestinales.

Bursera simaruba L. (almácigo)

La decocción de hojas y brotes se registra como anticatarral y febrífugo; de forma externa se aplican como cataplasma en el tratamiento de resfríados estomacales. La corteza tiene las mismas propiedades que las hojas, pero debe usarse con cuidado (sobre todo en los niños) porque es muy caliente. La aplicación local de resina es muy útil para extraer espinas de la piel.

Protium cubense (Rose) Urb. (copal)

La decocción de la parte aérea es anticatarral y antiasmático. La resina es útil en la extracción de espinas.

CACTACEAE

Nopalea cochenillifera (L.) Salm-Dyck (tuna blanca)

Las cataplasmas hechas con los tallos carnosos se consideran antiinflamatorios poderosos.

CANELLACEAE

Canella alba Murria (cúrbana, canelón, malambo)

La corteza macerada con alcohol se usa para fricciones antirreumáticas. La decocción cura las afecciones gastrointestinales y es un estimulante sexual. Tradicionalmente se brinda como condimento, anticatarral, febrífugo y afrodisíaco.

CAPRIFOLIACEAE

Sambucus intermedia Carr. var. insularis Schwerin (sáuco blanco)

La decocción de las flores es sudorífico y anticatarral y se emplea para tratar las infecciones oftálmicas. 


\section{CARICACEAE}

Carica papaya L. (frutabomba)

El jugo de la fruta se toma como digestivo y para tratar la difteria.

La decocción de la fruta tierna combate la diarrea infantil y las infecciones gastrointestinales. Las semillas y látex son vermífugos. La infusión de las flores se emplea como pectoral y febrífugo.

CECROPIACEAE

Cecropia peltata L. (yagruma)

La infusión de las hojas y los brotes se considera antiasmático, astringente y pectoral. El látex se usa para eliminar verrugas.

\section{CHENOPODIACEAE}

Teloxys ambrosoides (L.) Weber (apasote)

La decocción de la parte aérea, hervido en la leche, se considera un antiparasitario fuerte. El jugo es útil en lavados para curar las hemorroides.

\section{CLUSIACEAE}

\section{Calophyllum antillanum Britton (ocuje)}

La resina se registra como sudorífico y cicatrizante. La corteza, como colágogo para tratar las infecciones del hígado y como un estimulante para la membrana mucosa pulmonar. El aceite extraído de las semillas es bueno para tratar las afecciones de la piel y fortalecer el pelo; también se aplica localmente para curar infecciones del ombligo en los niños.

Mammea americana L. (mamey de Santo Domingo)

Se come la fruta cruda para tratar la anemia. La resina y la decocción de la corteza se aplican contra los parásitos, infecciones micóticas, y eczemas. La infusión de las hojas es un antifebrífugo.

Garcinia aristata (Griseb.) Borhidi (manajú)

La resina se toma como laxante, anticatarral, antiasmático y para extraer la frialdad del cuerpo. La decocción de hojas y tallos es útil para tratar las afecciones del aparato respiratorio como asma, bronquitis y pulmonitis.

\section{COMMELINACEAE}

Rhoeo spathacea (Sw.) Stearn (cordobán, barquito)

La decocción de la planta entera vía interna se considera anticatarral y sedativo, y es útil para tratar las irregularidades menstruales.

Zebrina pendula Schnizi (cucaracha)

El jugo obtenido de las hojas se aplica externamente como antihemorrágico.

\section{CONVOLVULACEAE}

Ipomoea batatas (L.) Lam. (boniato)

La decocción de la parte aérea se recomienda ampliamente como galactógeno. Una cataplasma del rizoma sirve para tratar las infecciones de órganos interiores.

\section{CRASSULACEAE}

Bryophyllum pinnatum (Lam.) Oken (siempreviva, prodigiosa)

Las hojas frescas ligeramente cocinadas en agua se aplican en las sienes para combatir las hemicranias. Se practican inhalaciones con la infusión de las hojas para curar la sinusitis.

\section{CUCURBITACEAE}

Citrullus vulgaris Schrad. (melón)

El cataplasma de la fruta se emplea como antiinflamatorio y su jugo desintoxica el organismo. Las semillas sin corteza son vermífugas.

Cucurbita moschata (Duchesne ex Lam.) Duchesne ex Poir (calabaza)

Se proponen las semillas como vermífugo. El jugo de las flores se aplica para combatir el dolor de oídos. 
Luffa cylindrica Roem. (estropajo)

Un enema hecho con el jugo de la fruta cruda es útil como vermífugo. La pulpa de las semillas se aplica de forma externa para eliminar piojos y sarna.

Momordica charantia L. (cundeamor)

La infusión de las hojas se toma como vermífugo, para tratar la colitis, los cálculos renales y las afecciones del hígado, además de regular la menstruación. Se toma el jugo de la fruta como vermífugo y laxante.

Sechium edule Sw. (chayote)

La fruta cruda o el agua de decocción se consumen como diurético. La pulpa cocinada se aplica como un cataplasma y es refrescante. La infusión de las hojas se toma para combatir la arterioesclerosis e hipertensión.

ERYTHROXYLACEAE

Erythroxylum havanense Jacq. (jibá)

Los tallos y hojas son buenas para el riñón y afecciones del hígado. La decocción de la raíz se emplea como antihemorrágico.

EUPHORBIACEAE

Abelmoschus esculentus (L.) Moench (quimbombó)

Un cataplasma con la fruta se considera antiinflamatorio. Se ingieren las semillas frescas como diurético y antiinflamatorio.

Jatropha curcas L. (piñón botija)

Se aplican cataplasmas hechos con las hojas para tratar los eczemas. El látex es un agente cicatrizante. El aceite extraído de las semillas es un laxante fuerte; muy tóxico también.

Jatropha gossypiifolia Lin. (tua tua)

La decocción de la planta entera, según informes recibidos, se toma contra las afecciones gastrointestinales y como un anticatarral. La infusión de las hojas puede ser emética o laxante, según si las hojas se han arrancado de la parte superior o bajo la mitad de la planta. Se considera tóxico.

Manihot esculenta Crantz (yuca)

El almidón tostado se emplea como talco en las erupciones de la piel en infantes.

Phyllanthus acidus Skeels (grosella)

Una infusión de las hojas se toma como sudorífico. Las semillas en la decocción son laxantes.

FABACEAE-CAESALPINIOIDEAE

Bauhinia cumanensis H.B.K. (bejuco de tortuga)

La decocción de hojas y tallos está registrada como antiasmática, anticatarral y para curar la bronquitis.

Caesalpinia bahamensis Lam. (brasilete, palo Brasil)

La decocción de la madera se toma para el tratamiento de las afecciones del hígado y los cálculos renales. Se considera diurético y depurativo y útil para tratar las úlceras crónicas.

Caesalpinia pulcherrima (L.) Sw. (guacamaya criolla)

La infusión de las hojas se ingiere para tratar las afecciones del hígado y se emplea como enjuague para eliminar las úlceras en la boca y garganta. La decocción de las flores se aplica en las afecciones de los ojos y como febrífugo.

Cassia grandis L. (cañandonga)

La pulpa de la fruta es ligeramente laxante y mezclada con leche y azúcar sirve para tratar la anemia. El jugo extraído de las hojas se aplica como antiherpético y otras infecciones de la piel.

Poeppigia procera Presl. (tengue)

La decocción de la corteza se utiliza para lavado de las heridas, infecciones por hongos y salpullido anal; es astringente, cicatrizante, antimicótico y cura las hemorroides.

Senna alata (L.) Roxb. (palo santo, guacamaya francesa)

Las hojas se han registrado como antiherpético y para lavados en el tratamiento de infecciones de la piel.

Senna obtusifolia (L.) Roxb. (guanina)

La decocción de las hojas se toma como laxante y la fruta y raíz como febrífugos. Se muelen las semillas con mantequilla y se aplica externamente para curar la sarna.

Tamarindus indica L. (tamarindo) 
La infusión de la fruta es febrífuga, estomáquica y sirve para curar las afecciones gastrointestinales, y la de la raíz es antihemorrágica y buena en las afecciones del hígado.

FABACEAE-FABOIDEAE

Andira inermis (Wright) DC. (yaba)

La decocción de la parte aérea se aplica externamente para curar las quemaduras causadas por el guao, y la de la corteza y semilla se ofrece como vermífugo y laxante.

Cajanus cajan (L.) Millsp. (gandúl)

Las semillas se aplican para tratar los flemones en las encías. La decocción de la flor y los brotes es expectorante.

Lonchocarpus domingensis (Pers.) DC. (guamá)

La infusión de las hojas es diurética,y la de la raíz, cortada en pedazos antes de hervirse, se indica para tratar la hiponuria y dificultad al orinar.

Mucuna urens (L.) DC. (ojo de buey)

La decocción de los tallos se toma como diurético.

FABACEAE-MIMOSIDEAE

Dichrostachys cinerea Wight et Arn. var. africana Brenan et Brummit (marabú)

La decocción de la corteza se aplica como cicatrizante y para tratar las infecciones micóticas, eczemas y hemorroides. Las semillas aplastadas y fermentadas se aplican como antimicótico. La infusión de la flor se toma como sedativo contra el insomnio.

FLACOURTIACEAE

Zuelania guidonia (Sw.) Britton et Millsp. (guaguasí)

La corteza puesta en agua se bebe como agua común para purificar la sangre. Si se pulveriza se aplica para curar las heridas. La resina es un laxante poderoso.

LAMIACEAE

Hyptis americana (Aubl) Urb. (orégano)

Se informa que la infusión de las hojas es anticatarral y útil en el tratamiento del dolor de oídos.

Hyptis suaveolens (L.) Poit. (orégano cimarrón)

La infusión de las hojas y los brotes florecidos se considera estimulante, estomáquico, purificador, y elimina los gases intestinales.

Mentha x piperita L. (toronjil de menta)

Una infusión de la parte aérea se toma para tratar el espasmo, regula la menstruación, eliminar los gases intestinales y como digestivo.

Ocimum basilicum L. (albahaca blanca)

La infusión de las hojas y tallos se bebe como un calmante, carminativo, estomáquico y bueno contra las diarreas. La decocción se aplica para fricciones en las piernas de niños y personas mayores para fortalecerlos.

Ocimum gratissimum L. (albahaca de clavo)

La infusión de las hojas alivia el dolor de cabeza y barriga.

Ocimum tenuiflorum L. (albahaca morada)

La infusión de la corteza es febrífuga y la de las hojas es sedativa y útil en la eliminación de los gases intestinales.

Origanum majorana L. (mejorana)

La decocción de la parte aérea se da como tónico y analgésico en los cólicos intestinales.

Orthosiphon stamineus Benth. (té del riñón)

La infusión de las hojas es útil en las afecciones de los riñones.

Pectis plumieri Griseb. (comino cimarrón)

La decocción de la parte aérea se toma como pectoral y antiasmático, y mezclada con miel es febrífugo y estimulante.

Plecthranthus amboinicus (Lour) Spreng. (orégano francés)

La infusión de las hojas se emplea para el tratamiento de la influenza y los resfríados. El jugo de las hojas se aplica para curar el dolor de oídos. Las hojas fritas se toman para curar la bronquitis.

Teucrium cubense Jacq. (agrimonia) 
La infusión de la parte aérea alivia las afecciones de la garganta. La decocción se usa externamente contra los edemas e hinchazón de las piernas.

Thymus vulgaris L. (tomillo)

La decocción de la planta entera se toma contra las afecciones gastrointestinales y dolores del estómago.

LAURACEAE

Cinnamomum cassia Blume (canela de China)

La corteza es un agente tónico, abortivo y útil para regular la menstruación. Mezclado con leche, huevos, y azúcar se toma como un ponche para tratar las afecciones gastrointestinales y contra la frialdad del aparato respiratorio. La decocción de la corteza se aplica en buchadas para curar el dolor de dientes.

Laurel nobilis L. (laurel)

La infusión de las hojas se toma como estomáquica y para curar el empacho. La misma infusión se aplica externamente como analgésico en las contusiones y en fricciones para tratar el reuma.

Persea americana Mill. (aguacate)

La masa del fruto cura las heridas infectadas. Además las semillas maceradas en agua son buenas contra la arteroesclerosis e hipertensión y la infusión de las hojas y brotes, para las afecciones cardíacas, artritis y para regular la menstruación.

\section{LILIACEAE}

Allium cepa L. (cebolla)

El bulbo se considera un antibiótico natural. En decocción o comiéndola cruda es: antiasmático, expectorante, y antidiabético. El cataplasma se emplea como cicatrizante, antiinflamatorio y analgésico. El jugo se aplica en las heridas; se considera antiséptico.

\section{LYTHRACEAE}

Lawsonia alba L. (resedá)

Se maceran las hojas en alcohol y se aplican en cataplasmas para curar el dolor de cabeza. La infusión de las hojas se toma para el dolor de barriga y la de las flores como sedativo. Si se toma como agua común es útil en el tratamiento de la hipertensión.

\section{MALPIGHIACEAE}

Byrsonima crassifolia (L.) H.B.K. (peralejo)

La decocción de la corteza se usa externamente para lavar heridas infectadas y en buchadas para tratar el dolor de muelas.

\section{MALVACEAE}

Gossypium barbadense L. (algodón)

Se dice que la infusión de la raíz regula la menstruación y facilita el parto. La infusión de hojas y brotes ayuda a la producción de leche en las mujeres.

Hibiscus elatus Sw. Majagua La decocción de las flores mezclada uniformemente con la salvia y el plátano, se emplea para tratar el asma y otras afecciones respiratorias.

\section{MARANTACEAE}

Maranta arundinacea L. (sagú)

La decocción del rizoma se emplea para curar ampollas e infecciones de la uretra. El almidón se considera nutritivo, digestivo, y antidiarreico.

\section{MELIACEAE}

Cedrela mexicana M.J. Roem. (cedro)

La infusión de la corteza se toma para tratar la epilepsia, inapetencia y ciguatera. La infusión de las hojas se dice es buena contra la tos y en buchadas para el dolor de muelas.

Guarea guidonia (L.) Sleumer (yamagua)

La decocción de la planta entera se conoce como el antihemorrágico más poderoso de Cuba. 
Melia azederach L. (paraíso)

La decocción de las hojas se emplea como febrífuga y antirreumática. De la raíz se informa como vermífugo.

Swietenia mahagoni (L.) Jacq. (caoba)

La decocción de la corteza se bebe como febrífuga y anticatarral y en buchadas para tratar el dolor de muelas y la piorrea.

Externamente, la decocción se usa en baños para las infecciones de la piel.

Trichilia glabra L. (siguaraya)

La infusión de las hojas es diurética y buena contra los cálculos renales. El jugo de la fruta en alcohol está indicado para fricciones antirreumáticas.

MORACEAE

Chorophora tinctoria (L.) Gaud. (fustete)

Las cenizas de la madera se utilizan para tratar la gota y las reumas. Con la decocción de la fruta se hacen gárgaras para las inflamaciones de garganta y boca. El látex combate el dolor de muelas.

MORINGACEAE

Moringa oleifera L. (palo caballero, paraíso francés)

Es antiasmático y útil en las afecciones de la próstata. La infusión de las hojas se toma para los dolores de músculos y huesos. Y la de las flores como sedativo suplente del tilo.

MYRISTICACEAE

Myristica fragrans Houtt. (nuez moscada)

Se brindan las semillas para tratar el dolor de cabeza y fortalecer la virilidad.

MYRTACEAE

Syzygium jambos (L.) Alston (manzanita lora, pomarrosa)

La decocción de las semillas pulverizadas se brinda para tratar la diabetes. Y de la corteza es un laxante poderoso y vomitivo. De la raíz es útil en el tratamiento de la epilepsia.

Pimenta dioica (L.) Merr. (pimienta dulce)

La decocción de hojas, tallos y semillas es antiodontálgico y afrodisíaco.

Pimenta racemosa (Mill.) J.W. Moore (pimienta negra)

Se aplican los cataplasmas de las hojas para curar los forúnculos infectados.

Psidium guajaba L. (guayaba)

La decocción de la fruta se toma para curar la diarrea; la de las hojas y corteza, se usa en baños para curar la escabiosis y heridas infectadas.

Syzygium aromaticum (L.) Merr. et Perry (clavo)

La decocción de la fruta y los tallos de la flor es útil como antiodontálgico y antiinflamatorio.

PAPAVERACEAE

Argemone mexicana L. (cardo santo)

El látex se aplica localmente para curar los herpes y eliminar las verrugas. Se ponen las semillas enteras en la oreja para curar el dolor de oídos. Las flores son pectorales y sedativas a dosis bajas, hipnóticas a dosis superiores.

PASSIFLORACEAE

Passiflora sp. (pasiflora)

La infusión de las hojas se considera sedativa y para combatir el insomnio. Un refresco hecho con la fruta es útil en la anemia.

PEDALIACEAE

Sesamum orientale L. (ajonjolí)

Las semillas son consideradas una comida nutracéutica por ser fuente de proteínas. De ellas se obtiene un aceite de 
propiedades antiasmáticas y antiinflamatorias.

PHYTOLACACEAE

Petiveria alliacea L. (anamú)

La decocción de la parte aérea es un antiinflamatorio útil en el tratamiento de las afecciones nerviosas y gastrointestinales. La raíz tiene las mismas propiedades pero más pronunciadas.

PIPERACEAE

Piper aduncum L. (platanillo de Cuba)

Una infusión de las hojas constituye un diurético poderoso y astringente. La decocción de la raíz se usa como hemostático.

Piper auritum H.B.K (caisimón de anís, anisón)

Las hojas frescas se aplican externamente para combatir las formaciones cancerosas. Se considera que la decocción tiene propiedades antiinflamatorias y diuréticas, es útil eliminando los gases intestinales. La infusión se brinda como refrescante y digestivo, pero puede ser muy tóxico en dosis superiores y si es ingerida por los niños.

\section{PLANTAGINACEAE}

Plantago major L. (llantén)

La infusión de las hojas se usa en gárgaras para curar el dolor de garganta y en buchadas para tratar las infecciones de la boca. Los cataplasmas son para combatir la diarrea y congestión del estómago (empacho) y también se hacen lavados vaginales para prevenir las infecciones. La decocción de las semillas se brinda como diurético, laxante y contra las hemorroides.

\section{POACEAE}

Bambusa vulgaris Schrad. (caña brava)

La infusión de las hojas es anticatarral, vermífuga y regula la menstruación.

Coix lacryma-jobi L. (Santa Juana)

La decocción de la planta entera se da en la cura de las afecciones respiratorias.

Cymbopogon citratus (DC.) Stapf. (caña de limón)

La decocción de los tallos es tónica y diurética, se da para combatir los cálculos renales, las inflamaciones pectorales y la gripe (frío, influenza).

Panicum purpurascens Raddi (yerba paraná, yerba del paral)

La decocción de la raíz, sola o con curujey y gandul, se administra para tratar la giardiasis y como vermífugo.

Sorghum vulgare Pers. (millo)

La decocción de la planta entera se usa como diurético y purificador de sangre.

Vetiveria zizanoides (L.) Nash (vetiver)

Se hacen fricciones con la decocción de la raíz para curar el reuma, artritis y neuralgias. La raíz se considera un insecticida.

Zea mays L. (maíz)

La infusión de la pelusa del maíz se toma para tratar las infecciones renales y los cálculos.

POLYGONACEAE

Coccoloba uvifera L. (uva caleta)

La infusión de las hojas es astringente y antidiarreica por vía interna y para curar las heridas, vía externa.

PUNICACEAE

Punica granatum L. (granada)

La decocción de la cáscara de la fruta se toma como vermífugo (contra Tenia sp.).

RHAMNACEAE

Colubrina arborescens (Mill.) Sarg. (bijáguara)

La decocción de hojas y tallos se emplea en baños para curar las afecciones de la piel, como mordeduras de serpiente y 
escabiosis.

Gouania polygama (Jacq.) Urb. (bejuco de indio)

La infusión de las hojas se da como diurético. Un baño con la decocción de hojas y tallos es bueno para las infecciones de la piel. La raíz se emplea para curar las heridas en la boca.

Gouania sp. (jaboncillo)

Las semillas secas y aplastadas se mezclan con el agua y se aplican para combatir piojos y escabiosis.

RHIZOPHORACEAE

Rhizophora mangle L. (mangle rojo)

La decocción de la corteza es astringente y cicatrizante, para tratar úlceras y diarrea. La maceración en el agua purifica la sangre, es antihemorrágico y febrífugo.

\section{ROSACEAE}

Prunus occidentalis Sw. (cuajaní)

Se informa que la decocción de la corteza es un antiasmático poderoso. El jarabe hecho con la pulpa de la fruta es ligeramente laxante.

\section{RUBIACEAE}

Calycophyllum candidissimum DC. (dagame)

La infusión de la parte aérea se da como febrífugo.

Chiococca alba (L.) Hitchc. (bejuco de verraco)

La decocción de la raíz es diurética, purifica la sangre y es afrodisíaco, pero a dosis superiores es laxante y emético.

Coffea arabica L. (café)

La infusión de las hojas es febrífuga.

Hamelia patens Jacq. (ponasí)

La decocción de hojas y tallos, externamente, se aplica para curar heridas y otras afecciones de la piel.

Ixora coccinea L. (Santa Rita)

La decocción de las hojas y tallos se aplica externamente contra las infecciones fúngicas. Se comen las flores como desintoxicante.

Morinda royoc L. (palo garañón)

Es afrodisíaco y estimulante sexual. La decocción de la raíz se considera un estimulante depurativo y sexual poderoso.

\section{RUTACEAE}

Citrus aurantium L. (naranja agria)

La infusión de la cáscara de la fruta se toma como sedativo, antidepresivo, febrífugo y para tratar las afecciones cardíacas.

Citrus limetta Risso (lima)

El jugo de la fruta se considera febrífugo y se aplica en los ojos para curar la conjuntivitis.

Citrus limon Burm. F. (limón)

La infusión de las hojas es anticatarral, febrífuga, y calmante.

Citrus medica L. (cidra)

Se toma el jugo de la fruta como estimulante y contra los espasmos. Su decocción se considera antiséptico y febrífugo.

Citrus paradisi Macf. (toronja)

El jugo de la fruta se toma como un antihipertensivo.

Ruta graveolens L. (ruda)

Se aplica jugo caliente de hojas y tallos para curar el dolor de oídos. La decocción es útil en el tratamiento de menstruaciones alteradas, hemorragias uterinas y para lavados uterinos.

SAPINDACEAE

Allophyllus cominia (L.) Sw. (palo de caja)

La decocción de hojas y tallos se brinda para tratar diabetes y afecciones gastrointestinales.

Blighia sapida Koen. (seso vegetal) 
La fruta aplastada se aplica como antiinflamatorio y febrífugo. La infusión de las hojas se toma para curar la congestión del estómago (empacho).

Cupania glabra Sw. (guarano, guarana)

Internamente es tóxico. En baños se brinda para curar infecciones de la piel, escabiosis y piojos. Las semillas se brindan como antidiarreico. La decocción de hojas y corteza se brinda para las afecciones gastrointestinales.

Melicoccus bijugatus Jacq. (anoncillo)

Se considera que la fruta cruda es antihipertensivo. La infusión de las flores se brinda como anticatarral.

SAPOTACEAE

Chrysophyllum cainito L. (caimito)

La decocción de la cáscara de la fruta se brinda como astringente y para tratar la diarrea. La pulpa se brinda externamente en las infecciones de la piel. Las hojas aplastadas se aplican para detener las hemorragias. La corteza pulverizada mezclada con la miel se ingiere como un febrífugo, y también las frutas crudas y el látex como febrífugo y diurético.

Manilkara zapota (L.) P. Royen (níspero)

La fruta se indica para tratar las aftas bucales y en gárgaras para el dolor de garganta. La corteza se pulveriza y se aplica en la frente como febrífugo. Las semillas, mezcladas con las del mamey colorado, se ingieren para curar la colitis y como diurético.

Pouteria campechiana (Kunth) Baehni (canístel)

La fruta ingerida se considera antianémica. El aceite obtenido de las semillas fortalece el cabello.

Pouteria sapota (Jacq.) H. E. Moore et Stearn (mamey colorado)

El aceite obtenido de las semillas se aplica para fortalecer el pelo y se ingiere para tratar colitis, diabetes y afecciones cardíacas. La savia se usa como emético y vermífugo.

SCROPHULARIACEAE

Capraria biflora L. (magüiro)

La decocción de la parte aérea se considera útil regulando la menstruación y en los fibromas uterinos. Las dosis elevadas pueden ser tóxicas.

Scoparia dulcis L. (escobilla)

La decocción de la planta entera se toma para tratar la diarrea y normalizar la menstruación.

SMILACACEAE

Smilax domingensis Willd. (raíz de China)

La decocción del rizoma es depurativa, sudorífica y antiasmática.

SOLANACEAE

Capsicum frutescens L. (ají picante)

La fruta es un agente antiinflamatorio fuerte y útil para aliviar la artritis. Se brinda contra las hemorroides pero también se informa que puede ser peligroso en la presencia de úlceras o fístulas anales.

Lycopersicum esculentum Willd. (tomate)

Las frutas se aplican externamente para curar las quemaduras.

Nicotiana tabacum L. (tabaco)

Las hojas se aplican en la frente para curar el dolor de cabeza. La decocción de la raíz se considera febrífugo.

Solanum americanum Mill. (yerba mora)

Una infusión de las hojas y los brotes se considera sedativo y narcótico; es antiséptico, antiherpético y sirve para curar las hemorroides.

Solanum melongena L. (berenjena)

Comer la fruta baja el colesterol en sangre y es bueno contra la arterioesclerosis. La infusión de las hojas se toma como sedativo.

Solanum tuberosum L. (papa)

Un cataplasma de las hojas se aplica para curar hemorroides. Los rizomas secos se aplican en la piel como un antiinflamatorio. 


\section{STERCULIACEAE}

Guazuma ulmifolia Lam. (guásima)

La decocción de hojas y corteza es útil contra la calvicie. Se come la fruta cruda en el tratamiento de anemia. El mucílago cura quemaduras causadas por tocar hojas de guao.

Theobroma cacao L. (cacao)

Se dan las semillas pulverizadas como tónico cardíaco y para curar infecciones en los riñones.

TILIACEAE

Corchorus siliquosus L. (malva té)

La decocción de la parte aérea se considera antiinflamatorio externa e internamente, y un febrífugo poderoso.

\section{TURNERACEAE}

Turnera ulmifolia L. (marilope, tapón)

La infusión de las hojas y flores es útil en el tratamiento en las afecciones gastrointestinales, como expectorante y estimulante.

\section{URTICACEAE}

Urera baccifera L. (chichicate)

La decocción de la raíz se brinda como diurético, purificador de sangre y eliminador de cálculos renales.

VERBENACEAE

Lippia alba (Mill.) N. E. Br. ex Britton et P. Wilson (quita dolor)

La infusión de las hojas y flores se toma como anticatarral, antiespasmódico y febrífugo.

Phyla scaberrima (Juss. ex Pers.) Moldenke (orozús de la tierra)

El jugo y las infusiones de las hojas se aplican como pectoral y estomáquico y para curar la bronquitis y tos.

Starchytarpheta jamaicensis (L.) Vahl. (verbena cimarrona)

La decocción de la parte aérea se da como depurativo, antidiarreico y útil en el tratamiento de patologías del hígado y alteraciones del ciclo de menstruación.

VITACEAE

Cissus verticillata (L.) Nicolson et Jar. (bejuco ubí)

La decocción de tallos y hojas se informa como sedativa. Un cataplasma de las hojas se aplica para tratar las inflamaciones.

ZINGIBERACEAE

Alpinia zerumbet (Pers.) B.L. Burtt et R.M. Smith (colonia)

Los baños hechos con la decocción de la planta entera son buenos contra las infecciones de la piel. La decocción del rizoma se considera diurética.

Costus speciosus (Koening) J.E. Smith (caña santa)

La infusión de las hojas se da como estimulante, diurético, anticatarral, y febrífugo. Las infusiones deben filtrarse por una tela para quitar la presencia de cristales en la parte aérea de la planta. Las infusiones mezcladas con el limón y las hojas de la naranja se toman para curar las afecciones del aparato respiratorio.

Zingiber officinale Rosc. (jengibre)

La decocción del rizoma se toma para tratar el reuma, como digestivo y para prevenir los vómitos y náuseas, como un afrodisíaco y para nivelar los altos niveles de colesterol en la sangre. La decocción mezclada uniformemente con el sasafrás y cúrbana también se emplea en fricciones contra el reuma y artritis.

\section{ZYGOPHYLLACEAE}

Guaiacum officinale L. (guayacán)

La corteza triturada se aplica cuando hay dolor de muelas como anestésico. 